\title{
A Systematic Review: Using Habits of Mind to Improve Student's thinking in Class
}

\author{
Suad Alhamlan ${ }^{1}$, Haya Aljasser ${ }^{1}$, Asma Almajed ${ }^{1}$, Haila Almansour ${ }^{1} \&$ Nidhal Alahmad $^{2}$ \\ ${ }^{1}$ Curriculum \& Instruction Department, KSU, Riyadh, Saudi Arabia \\ ${ }^{2}$ Professor in Science Education, Department of Curriculum and Instruction, King Saud University, Riyadh, \\ Saudi Arabia \\ Correspondence: Suad Alhamlan, EFL supervisor, Ministry of Education, Riyadh, Saudi Arabia. E-mail: \\ suadalhamlan@gmail.com
}

Received: December 11, 2017 Accepted: December 29, 2017 Online Published: February 6, 2018

doi:10.5539/hes.v8n1p25 URL: https://doi.org/10.5539/hes.v8n1p25

\begin{abstract}
This review article aims to explore how habits of mind as the concept defined under the recent Framework for Success in Postsecondary Writing contributes to the development of critical thinking in the classroom. The application of a systematic review and a meta-analysis approach allows investigating the selected habits of mind and comparing them to the externally verified critical thinking skills using a multiple regression with a variation methodology. Study results suggest that the available literature does not provide a comprehensive analysis of the problem due to the variations in explanatory values of the variables selected; though, it justifies the instructional leadership approach that should be fulfilled by the educators striving to deploy the Framework in their educational environment. The review suggests using a fieldwork approach as the next step of the mentioned Framework evaluation.
\end{abstract}

Keywords: habits of mind, critical thinking, mind theories, motivation, student engagement, learning theories

\section{Introduction}

\subsection{Statement of the Problem}

The importance of student's critical thinking skills development has been broadly discussed by researchers and reinforced by accrediting bodies in the educational facilities. A general view of the problem suggests that the students who have been actively engaged into critical thinking activities during high school years are more adapted to college and university education, as well as smoother integrated into a working environment afterwards. This view is also supported by the theory of student engagement, which postulates that the higher the social and academic connection of the student to the respective educational facility, the higher the likelihood to demonstrate his or her persistence in learning efforts (Jacobson, 2014). However, the factors attributable to critical thinking development remain understudied, suggesting several broad perspectives that require further investigation to justify them.

One of those factors recently discussed by academic research and introduced by several educational frameworks is known as the habits of mind. The recent Framework for Success in Postsecondary Writing jointly developed by the Council of Writing Program Administrators, the National Council of Teacher of English, and the National Writing Project in January 2011 (further, Framework) stated that readiness for college entrance should be defined not by specific standards but by intellectual behaviors and educational experiences that encompass rhetorical skills as well as habits of mind (Johnson, 2013). The Framework indicated that the critical habits of mind that could be fostered through reading, writing and critical analysis are creativity, openness, curiosity engagement, persistence, flexibility, responsibility and meta-cognition (O'Neill, Adler-Kassner, Fleischer, \& Hall, 2012). These habits also frame a learning approach from both intellectual and practical aspects, assuming that students should demonstrate adherence to both learning curriculum and developing themselves externally. Moreover, it was stated that teachers should engage learners into reading texts from multiple viewpoints and being able to evaluate those texts from credibility, author's bias and quality of reasoning standpoints (How \& Wig, 2017). On the other hand, it was stressed that under the habits of mind concept, it is critical to write about previous reading experiences from multiple purposes, including interpretation, synthesis, and responses articulation (O'Neill et al., 
2012).

The adoption of the aforementioned standards in postsecondary education requires structured and complex efforts from both students and teachers, as well as extensive support from accrediting organizations. Specifically, it is critical to provide a guidance and benchmark for implementing and measuring an approach based on the habits of mind thinking. A scale for evaluating learning behaviors that measures information reliability evaluation, outdoor exploration efforts and alternative problem-solving was proposed as an outcome of the Cooperative Institutional Research Project. However, several authors suggested the scale was not much adopted for initial institutional changes since there is a gap of understanding student's critical thinking skills as well as the lack of previous surveys on how teachers contribute to such self-assessment efforts (Jacobson, 2014; O'Neill et al., 2012).

\subsection{Research Objectives}

Considering the need of the improved strategies to adopt the new framework into a postsecondary education on the one hand, and the need to establish effective benchmarking and assessment tools from measuring the success of it on the other hand, this research will consider recent findings from the individual studies conducted in the field of habits of mind application to the educational programs. The primary objective of the study is to identify what habits of mind adjacent to the Framework contents contribute to the development of critical thinking in class among postgraduate students considering the complexity of disciplines requiring critical thinking skills and the explicit need of multidisciplinary thinking in a future business environment. The secondary objectives are to analyze the efficiency of the current educational system in assessing and implementing those efforts through the methods proposed in those studies, and to suggest possible steps for the improvement of those efforts.

\subsection{Research Questions}

The research comprises a theoretical part, where the backgrouding to the habits of mind formation is established by elucidating the sense of the habits of mind from learning theories and comparing it to the current view of the literature, and practical part, where the statistical analysis methods are used to validate the relationships between the variables that describe the habits of mind and the variable that explains critical thinking skills. A systematic review through a meta-analysis approach is used as a methodology, hypothesizing that the habits of mind within the Framework and critical thinking skills of student's demonstrated in class are positively related. The study contributes to the existing literature by providing an expanded view on using habits of mind for critical thinking improvement theoretically, and suggesting a pathway for schools and accrediting bodies to deploy the Framework practically.

\subsection{Research Gap and Importance of the Problem}

The research addresses the gap that exists in a current transition from the standard requirements for colleges and universities to accept postgraduates based on the standard procedures to the requirements of considering educational experiences and intellectual behaviors demonstrated in class. This requirement also intersects with the need of multidisciplinary education that will address social and economic aspects of emerging professions and specialties that accumulate various experiences of creative, technical and interactive nature (Kleinsteuber, 2014). Last but not least, present literature does not provide much reliance on the existing learning theories, which place a concern on whether they should be applicable for new educational framework development or must be re-mapped to meet the demands of business and changing learning curriculums in higher education.

\subsection{Theoretical Background}

\subsubsection{Learning Theories and Habits of Mind}

The concept of habits of mind is emerged from the field of brain research and education. This concept entails the habits as the behaviors that could be used appropriately without painstaking attention, or requiring some brain activity that was not initially adopted (Gardner, 1983). Habits are described as almost instinctive behaviors that provide mental space for problem solving while not requiring recalling a specific type of thinking to be used. Habits were also defined as a set of dispositions learned and practiced before an individual can accomplish a specific task set almost effortlessly (Costa \& Kallick, 2000). Habits were further related to the research on learning and intelligence so that the historical context of habits of mind is explained. While Perkins (2000) explained that habits are defined through continual practice, Costa \& Kallick (2000) expanded this definition to the habits of mind as series of intellectual behavior patterns that could lead to the demonstration of productive behavior. In their further works, Costa \& Kallick (2009) conceptualized 16 habits that could equip students with means to make more intelligent choices both within and outside the classroom environment. Some of the examples listed are questioning and posing problems, applying past knowledge to new situations, finding humor 
or taking responsible risks. The habits of mind were applied to many classroom setting examples; though, they were not meant to be proposed as a replacement to the existing learning processes (Costa \& Kallick, 2000; Costa \& Kallick, 2009).

Another origin of the concept could be found in the multiple intelligence theory that was concerned with defining linguistic and logical-mathematical use of intelligence measurements based on the brain damage and cognitive analysis proceedings of the scientific literature. Initially proposed by Gardner (1983), this theory explained that the intelligence should be defined by much broader and modular terms using eight distinct signs (i.e. support from psychometric findings or a brain susceptibility to a symbol system encoding), resulting into defining seven types of intelligence. Criticizing the theory for many misconceptions, it was further combined with the theory of mind and formulated as the theory of whole-mindedness, using the recent proceedings in cognitive neuroscience (Groff, 2013). The main aspects outlined in a new theory is that intelligence should be associated with certain learning domains while not positioned as the distinct domain type not intersected with other domains. It was also specified that multiple intelligence theory tends to mix intelligence with learning styles, labeling each student as a certain type of learner (Groff, 2013). To address those misconceptions, the theory suggested seeing the learning process not through types of intelligence but through the non-verbal information processing pathways. According to Groff (2013), these pathways comprise the visual-object pathway that recognizes shapes, colors, and textures, the visual-spatial pathway that is applicable to spatial relationships in natural sciences or the movie production industry, and the text-based pathway that uses the language to transmit information and is most frequently used in education. Considering the neuro-scientific research, Groff (2013) pointed out that all learners are capable to possess all three information processing pathways, while learning acquisition normally shapes those to a single pathway. This was later confirmed by the web-based study conducted by the Group Brain Project that clustered over 80\% participants being strong non-verbally, and about 50\% as object-visualizers among the sample of 3,800 participants.

\subsubsection{Student Engagement and Critical Thinking}

The extent of student involvement in learning that motivates one to perform with a certain success is described by several learning theories. One of those theories, the student engagement theory, postulates that persistence demonstrated by students, especially in their first years of study, relates to the level of further involvement into educational process (Astin, 1999). The theory emerged from three other concepts, such as the subject-matter theory favored by the faculty, the resource theory favored by administrators, and the individualized-eclectic theory favored by researchers, and provided the means to quantify the notion of students' motivation and set of specific behaviors. Previous research also stated that the student engagement theory helped to make a shift from the mass to universal higher education, where the skills of non-traditional student (i.e. those participating in student exchange programs or students demonstrating non-traditional thinking patterns) are comprehensively evaluated.

Critical thinking is one of the concepts explored under the student engagement theory, while the concept itself had been debated even earlier. In his work, McPeck (1984) argued that critical thinking requires complete disciplinary expertise, which means that reasoning skills and argument-based analysis are not related to critical thinking. In their turn, cognitive psychologists related all forms of problem-solving, evaluating, and decision-making to critical thinking (Williams, 1999). Later definitions also conceptualized critical thinking as information gathering, questioning in self-reflection practices that lead to expressing self-generated ideas and conclusions (Weiler, 2005). Hence, it was later summarized that critical thinking could not be taught on its own and should be seen as a skill developed separately from the disciplinary content (Hammer \& Green, 2011).

The problem related to developing critical thinking skills within the learning curriculum was described by Willingham (2008), who contended that creative problem solving is not possible outside the disciplinary knowledge, since both content and critical thinking occur simultaneously. The researcher also admitted that students do not transfer the expertise easily, as, for instance; a student might of solving a math exercise but will have difficulties of applying the same algorithm to solving a real-life problem. Hence, it is clear that people are unable to become critical thinkers overnight, which means that the development of critical thinking should be seen as a meta-cognitive strategy that emerges from specific disciplines, and if used over time, could be mastered and possibly transformed into the multidisciplinary expertise (Jacobson; 2014; Willingham, 2008).

\section{Literature Review}

\subsection{The Role of Habits of Mind for Critical Thinking}

In more recent research, it was argued that the thinking indicators outlined above could be labeled as the habits of mind, and could be acquired through a developmental approach that increases the likelihood of successful 
student performance. Ahuna, Tinnes and VanZile-Tamsen (2011) suggested dividing them into meta-strategic, meta-cognitive and epistemological behaviors, which stand for knowing how, knowing what, and how do I know respectively. A meta-strategic dimension is discussed with stronger importance to the education field in early years of study in particular, as its understanding allows the instructors and curriculum developers to create a favorable environment for critical thinking efforts as the time passes (Jacobson, 2014). This claim is supported by a practical study undertaken by Walker, Wartenberg and Winner (2013), who reported that there are improved augmentation skills and increased subjectivity perception among seven and eight year olds exposed to a dialogic learning model. Supporting the development theory of critical thinking, Jacobson (2014) suggested that the aforementioned approach supports an assertion that behaviors from particular habits of mind are examples of a meta-strategic dimension, while there is a certain literature gap in understanding whether such an assertion holds for student-level thinking.

Reverting back to the case of how critical thinking relates to the disciplinary paradigm, it is essential to consider that various disciplines should be associated with various habits of mind and hence differentiated in terms of individually emerging critical thinking models. For instance, Kleinsteuber (2014) investigated how professional development in the area of arts is influenced by specific habits of mind combined with teacher self-efficacy through an exploratory mixed-method approach. Using the framework presented by Sheridan (2011) that outlines the studio habits of observing, envisioning, exploring and reflecting, Kleinsteuber concluded that there is a high correlation between the studio habits and teacher's self-efficacy, as well as the factors that mediated a decision to participate in arts education. On the other hand, it was found that prior art experiences have a little significant correlation with the studio habits and teaching skills applied. To some point, this contradicts previous theoretical assumptions on the necessary disciplinary activity as a prerequisite for habits of mind development.

Matsuura, Sword, Piecham, Stevens \& Cuoco (2013) explored another important discipline with respect to the habits of mind development. The researchers explored the importance of the habit of using mathematical language, which is about thinking, mental habits and research techniques applied to the explanation of more complex terms, such as definitions, theorems, and algorithms. Their investigation pointed that it is significant that teachers could learn how to transfer such knowledge to the students, especially on the secondary level. Given that the study has a preliminary essence and was not based on any previous empirical studies but rather proceedings of mathematical learning communities, it should be extended and improved with further research to bring more discourse to this point of learning.

King (2013) also discussed the importance of developing mathematical habits of mind among students. In her work, the researcher argued that productive habits of mind in this direction are complex to develop given that students are frequently trying to approach the tasks in abstract algebra by attempting to look for a quick answer, not being persistent when the answer is not obvious and tending to memorize the approach but not to understand it. The important question raised by King (2013) is whether a contemporary approach of teaching mathematics indeed teaches critical thinking or maybe, it provokes students to use bad habits of mind to ignore the difficulties emerging on their way. However, King (2013) has not provided any methodological evidence to support this claim; hence, this research requires further justification using field investigation.

Many other recent researchers focused on specific habits of mind as they have been described under the Framework. For instance, Sullivan (2012) reflected on the importance of habits of mind as a prerequisite for college readiness, focusing on the skills of curiosity, openness, creativity, accountability, and humility. Having the first four skills on the Framework's list and the last not, Sullivan (2012) argued that it is imperative to include humility as the habit of disposition towards the world, intellectual work, and production of meaning in one's lives. Overall, Sullivan (2012) concluded that dispositional qualities and personal traits should be clearly accepted by students against the purpose of scoring the passing grade in entrance tests; while further qualitative research undertaken would have significantly improve the researcher's opinion on the subject.

The importance of the habits of mind was also discussed with reference to the technical disciplines that foster further understanding of applied engineering tasks. The National Academy of Engineering (2010) proposed three general principles as they apply to the K-12 standards of engineering education - the emphasis of design in engineering, the incorporation of developmentally appropriate skills in mathematics, science and technology, and the promotion of the engineering habits of mind. Reflecting on the last point, Loveland and Dunn (2014) proposed to embed the innovative approach into the secondary education by offering students different design challenges that could contribute to the formation of the relevant knowledge base before the post-secondary education. The examples included the cost analysis of production process, exploring the effects of oil spills on the fish population in the water, or building series and parallel electric circuits for innovative technology solutions, such as light LEDs. By narrowing down the focus for a particular skill, Lammi and Denson (2017) 
discussed the importance of modeling as an engineering habit of mind, suggesting the modeling artifacts in conceptual, graphical, mathematical and working representation that could be used in a classroom setting. After having applied this framework into a real situation with a group of students, they concluded that the framework helps to demystify that the engineering process is linear, allowing to approach the tasks with not only a direction and guidance but also with ability to model engineering solutions on their own. However, Lammi and Denson (2017) admitted that a more extended review could be required to check the applicability on pre-college students in various years of study, signifying that modeling is a more advanced critical thinking exercise if applied to the secondary studies.

\subsection{Research Hypothesis}

The reviewed literature points on the significant efforts in exploring how the habits of mind contribute to the formation of critical thinking skills. There is an obvious connection to learning theories presented by scholars in the future, while several changes and adoptions have been made to shape the understanding of the importance of diversifying disciplinary thinking from critical thinking. Moreover, little studies explored the impact of the habits of mind classified under the Framework, otherwise listing them as applicable for several disciplines taught in the classroom and arguing on the needs to add one or another dimension while not providing a methodological rational behind. Nevertheless, the research hypothesis is to explore if there is a positive relationship between the habits of mind listed under the Framework as predictors of improved critical thinking demonstrated in class.

\section{Method}

\subsection{Research Design}

The information from the recent publications related to the aspects of the habits of mind and their applicability to critical thinking is analyzed using a systematic review and a meta-analysis approach. The chosen approach has several advantages comparing to the previous studies. The first advantage is that it was found that previous research was cross-disciplinary, since the articles were published in many scientific journals in the fields of ethics, education, cognitive psychology, and applied sciences, such as engineering and mathematics. Hence, organizing this information into a systematic review helps addressing the gaps that have been probably overlooked while narrowing the study to specific field of research (Ellis \& Murtha, 2014). The second advantage is that the application of systematic review allows comparing effect size across several variables, when samples are not comparative if two methods are just equated (Fisher, 2017). The last one is that many literature pieces found on the subject are classified as gray under research methods, given that unpublished statistics and recent dissertations are included. Hence, a more balanced and accurate view of the findings could be presented.

The choice of a meta-analysis has also its advantages under the scope of the current study. In case of the reviewed literature on the impact of habits of mind on critical thinking in the class, a meta-analysis allows maximizing the precision of variance if a predictor is explored (Fisher, 2017). Further, literature suggests that quantitative exploration of the source of heterogeneity is possible when using the meta-analytical methods, which enables more transparent hypotheses testing (Petinou \& Spanoudis, 2014).

\subsection{Sample}

\subsubsection{Sampling Strategy}

The sampling method adheres to the procedure outlined in PRISMA protocol for conducting systematic reviews (Higgins \& Green, 2008; Liberati et al., 2009). A prior literature research was conducted in electronic databases such as JSTOR, BASE, EBSCOSearch and ProQuest, placing no limit to the year of publication. The search terms used during this activity where sourced from the proposal to the project review and consultation with a direct supervisor. The search request in the web browser looked as follows: (*habits of mind* OR *mind theory*) AND (critical thinking) AND (education). Additionally, citations to the studies that met the inclusion criteria were reviewed with respect to the learning theories and reference to the subject. Finally, statistics and reports on the Framework adoption were reviewed, seeking for essential figures and justification.

\subsubsection{Sample Size, Power, and Precision}

The initial objective was to collect at least 150 pieces of the prior literature to meet the criteria of sufficient sampling under the meta-analysis requirement. The final search yielded 103 objects that met the search criteria. At the second stage, the inclusion and exclusion criteria were applied to the pieces of literature pooled from the database.

The inclusion criteria were applied as follows: (a) research participants were secondary students or the sample included secondary students, (b) the study was prospective in design and assumed either review of the existing 
methods or perspectives for further analysis based on an innovative approach, (c) optionally, the study should have related to the Framework, (d) the study is available in English.

The exclusion criteria were applied as follows: (a) participants are teachers only or postgraduate students only, (b) participants are affected by specific circumstances (i.e. adoption, having disabilities or undergoing mental treatment), (c) the study had an exploratory nature and did not list any methodological section, (d) the predictor was not in line with the research question and/or unfavorable for meta-analysis. As a result, the research precision was narrowed down to the 27 pieces that meet both inclusion and exclusion criteria.

\subsubsection{Sampling Validity}

The primary outcome measure selected to display and validate the scores for the selected habits was borrowed from the methodological approach used by Kleinsteuber (2014) as the study used the most relevant methodology. Other studies were explored for the variables similar to those from the habits of mind used by the primary source. The critical thinking scores were obtained from another primary source (Jacobson, 2014) while also validated against the Framework proceedings outlined by O'Neill et al. (2012) and the statistics collected from the Framework deployment reports from the anonymous source. The covariates were not included.

\subsubsection{Methods of Data Collection}

The research design is a multiple regression model, which uses five independent variables as predictors (gathering all data through senses, thinking flexibly, persisting, thinking and communicating with clarity and precision, and metacognition) and the critical thinking rate as a dependent variable. Predictors are adjusted as per the scale used in Kleinsteuber (2014), while the dependent variable is adjusted as per the Likert scale, where ' 1 ' stands for not sufficient, ' 2 ' stands for developing, ' 3 ' stands for optimal, ' 4 ' stands for very good, and ' 5 ' stands for outstanding. The model is presented below:

$$
E(y)=\beta_{0}+\beta_{1} x_{1}+\beta_{2} x_{2}+\beta_{3} x_{3}+\beta_{4} x_{4}+\beta_{5} x_{5}+\varepsilon
$$

where $\mathrm{x} 1 \ldots \mathrm{x} 5$ are independent variables (predictors) and $\mathrm{E}(\mathrm{y})$ is the dependent variable as listed above. The objective was to identify the model significance, the justification of the impact of each variable over critical thinking, both individually and overall, as well as to find the variation and to justify possible heterogeneity effect.

\subsubsection{Sample Setting}

Considering the nature of systematic reviews and meta-analyses as outlined by Liberati et al. (2009), there was an allowed extent of manipulation to standardize the data before completion of the analysis part. The essence is to use the same numbers for similar habits of mind, adjusting them to the common representation under the framework. Table. 1 shows the approach replicated from Kleinsteuber (2014), which explains how the process was undertaken.

Table 1. Manipulations and explanations for the habits of mind used

\begin{tabular}{lll}
\hline Systematized Habits & \multicolumn{2}{c}{ Habits of Mind (Framework) } \\
\hline Observation & Gathering data with senses & Listening with understanding \\
Envisioning & Creating, imagining and innovating & $\begin{array}{l}\text { Applying previous knowledge } \\
\text { to unfamiliar situations }\end{array}$ \\
Stretching and Exploring & Thinking flexibly & Taking reasonable risks \\
Expressing & $\begin{array}{l}\text { Thinking and communicating } \\
\text { clearly and precisely }\end{array}$ & $\begin{array}{l}\text { Responding with wonderment } \\
\text { or finding humor }\end{array}$ \\
Understanding the World of Experience & Being open to continuous learning & Interdependent thinking \\
\hline
\end{tabular}

\section{Results}

\subsection{Timeframe}

Pieces of literature were analyzed during the first month of the research. The adjustment to the secondary sources and the consolidation with statistical input was conducted during the second month of the research.

\subsection{Statistics and Data Analysis}

A separate, random-effect model was considered as the most representative outcome of the statistical analysis for each predictor using the SPSS software. The acknowledged confidence interval was 95\%. Cohen's (1988) conventions for interpretation of multiple regression models were considered when the sample size is small $(r$ $=.1)$, medium $(r=.3)$ and large $r=.5)$. The main effect of meta-analysis was reported through relationships 
between continuous predictors and outcomes using a linear relationship model. The statistical significance of heterogeneity was explained within the model provided by Borenstein, Hedges, Higgins, \& Rothstein (2009) and further with interpretation of the I square using Higging \& Green (2008) model.

Table 2. Data analysis results

\begin{tabular}{|c|c|c|c|c|c|}
\hline \multirow[t]{2}{*}{ Predictor } & \multirow[t]{2}{*}{ Studies } & \multicolumn{2}{|c|}{ Main effect } & \multicolumn{2}{|c|}{ Heterogeneity } \\
\hline & & Pearson's $r$ & $95 \% \mathrm{CI}$ & p-value & $\overline{\mathrm{I}^{2}}$ \\
\hline Observation & 12 & 0.241 & {$[.123, .289]$} & 0.039 & 46.3 \\
\hline Envisi & 21 & 0.045 & {$[.035, .046]$} & 0.176 & 29.5 \\
\hline Stretching and Exploring & 14 & 0.231 & {$[-.039, .041]$} & 0.281 & 19.4 \\
\hline Expressing & 16 & 0.023 & {$[.021, .027]$} & 0.101 & 35.2 \\
\hline Understanding the World of Experience & 8 & 0.865 & {$[.541, .923]$} & 0.131 & 33.7 \\
\hline
\end{tabular}

Data results could be interpreted as follows. Firstly, by considering the Pearson's correlation coefficient, the most significant results are related to envisioning and expressing, while observation, stretching and exploring, as well as understanding the world of experience are seen as not significant. Secondly, the heterogeneity shows that the multiplication effect of the studies considered for a meta-analysis suggests non-dependence from the multi-collinearity of variables only in terms of observation, whereas the rest of variables could be affected by differences in other representations. The overall reported strength of the model also shows that the coefficient of determination ( $r$ squared) is 57\%, which means that only 57\% of variation is explained with the independent variables, and the rest $43 \%$ requires additional explanation through the residual points if identified in the consequent trial of runs for multiple regression model evaluation. The result for the regression model itself is also relatively weak, since it shows the overall insignificance of the factors as they are compiled for a single use, which is yet to be explained with reference to the previous works and possible research enhancements.

\section{Discussion}

\subsection{Empirical Results and Comparative Analysis}

A consolidated view through a systematic review and a meta-analysis of the statistical data collected through inclusion and exclusion from the subject matter shows that the selected habits of mind have a varying effect on critical thinking among students in the classroom. This is justified with differences for multiple regression results and corresponding heterogeneity effects as they appear in the results for various habits and finding pertinent to the research objectives. Previous considerations on such inconsistency derive from the methodology used within such purposes, while several explanations are required on the side of the other literature and theory applications.

Previous literature suggested considering the role of instructional leadership as the driving principle for educational reforms, which is applicable to the case of deploying the habits of mind reflected as not sufficient according to the results obtained (Augustine, 2014; Chahine, 2011). Student-centered focus on learning is mainly enforced by instructional leadership as well. Generally, instructional leaders perform the same role as the teaching facilities, like the most efficient contributors to the learning curriculum. However, studies in social and educational theories emphasize on the differences on how instructional leadership forms should be conceptualized. Rigby (2014) mentioned that both the institutional and external environments should be considered when principals determine the extent of engagement among instructional leaders. The way how instructional leadership should be deployed in order to facilitate the habits of mind integration into the critical thinking skills development depends on the learner's group, subject, and particular learning objectives. Among the above-mentioned, Tolley, Johnson \& Koszalka (2012) outlined generating and testing hypotheses, comparing and contrasting specific cases, summarizing and organizing information into sections, as well as note-taking. Hence, it could be said that the essence of instructional leadership is to create parallels for human brain work, which requires learners to be focused on a particular content through a defined learning period, engage students into decision-making and problem-solving, and set individual performance goals, like choosing particular research projects on their own. In other words, instructional leadership encourages students to actively participate in their own educational process by using strategies that conform to the biological nature of human brains.

In line with the Framework requirements, instructional strategies in the learning process should focus on the transformation of learning experiences through challenging cognitive learning models, freedom and empowerment of thinking, hence reframing a learning approach in secondary education to the self-centered efforts (Rigby, 2014; Haber-Curran \& Tillapaugh, 2015). The designated framework of this transformation is 
student centered-learning - a process where "students learn to be critically reflective and examine their own beliefs" (Haber-Curran \& Tillapaugh, 2015, p. 67). Such a principle shows much relevance to deploying critical thinking skills as through student's-centered engagement into the habits of mind development. The importance of engaging students comes from a common vision on the crossroads of social sciences, educational systems and an economic situation to develop students that will be capable of significant contribution to the community, where their skills and knowledge are valuable and relevant (Gershman, Gerstenberg, Baker \& Cushman, 2016). Student-centered learning, just as the instructional strategies, appears in many forms, and could be relevant in one case but completely irrelevant in the other. For instance, a cogenerative dialogue as a form of student-centered learning, which allows using dynamics occurring within the classroom as practicing one's leadership skills, might be a challenging form of knowledge acquisition in the classrooms where leadership perceptions were not prior cultivated by the instructors (Stith \& Roth, 2010).

Academic literature suggests that student-based learning could be introduced by shaping teacher-based learning through transformation driven by instructional leadership (Fierke et al., 2014; Haber-Curran \& Tillapaugh, 2015). In the meantime, instructional leadership would appear in different forms depending on the external conditions and the institutional environment, which means that transformation should occur through "connecting the dots" between the logic of instructional leadership and principles and forms of student-centered learning. Rigby (2014) defined three forms of logic applicable for instructional leadership in the context applied to the principles. The first type is prevailing logic based on the perception of the educational facility principal as both an instructional leader and a manager. It means that the scope of principal's roles somewhat expands those of organizational managers, like establishing relations with subordinates (teachers), guide and supervise them to better perform their work (improving instruction capabilities towards the increased achievements among students) and maintain focus on learning and instruction as the key role responsibilities (Rigby, 2014). This logic represents a shift from traditional way of school management in the previous century, where principals were framed only within the educational context rather than social or organizational ones. This approach was incorporated in the set of standards at the government level, covering principal preparation programs and evaluation standards called the ISLLC standards (Glenewinkel, 2011).

The second type of logic is entrepreneurial, which, according to Rigby (2014), "promotes education reform through activities, beliefs, and mechanisms borrowed from the private sector" (p. 623). Entrepreneurial logic is, though, defined as an opposite to public, meaning that various technological, innovative or borrowed practices would dominate in the principal's mindset rather than following practices already adopted and acknowledged by teaching community. Comparing entrepreneurial logic to prevailing, Rigby (2014) suggests that instructional practices for the former are research-based, while the latter will rely on teaching experience and specific teaching practice differentiated from one teacher to another. The role of instructional leader guided by entrepreneurial logic, though, is to "bring outcomes-focused curricula to teachers" and "enforce/support/monitor use of such curricula" rather than leading teachers towards a vision of students' achievements (Rigby, 2014, p. 625).

The third type of logic analyzed by Rigby (2014) is social justice logic, which promotes critical thinking and preparedness for the inequality challenges among student groups. The ultimate role of instructional leadership employed by principals in this logic is to change beliefs, thus facilitating extended access among student group to the rich and engaging curriculum that would satisfy the condition of social justice upon their graduation. By social justice, despite its broad definitions, here we assume the existence in the society that promotes equality, especially for groups considered historically marginalized (Rigby, 2014). Other than that, social justice logic calls for developing proactive attitude among students through their engagement in self-education, which is also a norm of student-centered learning.

According to Rigby's (2014) research, transformation through instructional leadership should start by rethinking the prevailing logic towards social justice logic, where the broad goal of student achievement and teacher satisfaction is narrowed to the engagement of students towards self-realization based on the principle of the equal opportunities. Emerging trends in these directions also conform to the need of multidisciplinary thinking as it was stated in the initial suggestion of embedding habits of mind to the notion of critical thinking skills in the classroom. Haber-Curran \& Tillapaugh (2015) suggested a model that explains how student's attitudes and beliefs would change as a result of appropriate change in the instructional leadership methods combined with the habits of mind. Their process of student's learning and relationships starts from the challenging of mental models of learning, gradually advancing the state of reframing learning and self through building trust, finding freedom and cultivating commitment to learning. In other words, Haber-Curran \& Tillapaugh (2015) demonstrated that students guided towards student-centered learning undergo the mental transformation of dependence/independence towards the interdependence, where self-motivation to learn is acquired through an 
understanding of the importance of knowledge acquisition for future success and equal place of teachers and students in their efforts of knowledge cultivation. This observation is also confirmed adversely by an applied study by Tolley et al. (2012) for Thai school students. The researchers concluded that "implementation of research-based instructional strategies intended to improve students' level of engagement had an impact on teaching practice for this group of teachers" (p. 391). Similarly, Zhu and Engels (2014) confirmed that organizational culture applied to the context of institutional environment has an impact on instructional innovations, facilitating the development of new teaching tools and methodologies.

\subsection{Limitations}

While the theoretical part assumes that research findings withhold, the review has its limitations. First, despite the benefits of a systematic review and a meta-analysis approach, it is essential to highlight that the statistical method used for analyzing the results is imperfect, as it allows replicating previous findings without appropriate justification of methods to simplify an approach for reasoning. This could be possible avoided with broader availability of the research on the subject related to the Framework positioning, while given it reference and innovative subject for the academic purpose, it leaves a limited choice for extrapolating other views and make other inputs. Second, the review was aimed towards the dissemination of meanings for various disciplines, while it mostly highlights the applicability of disciplinary attitude that fosters the culture of self-development, possibly more relevant to the management studies rather than an educational review. Last but not least, there is a lack of available institutional approaches that are comprehensively reported in the electronic libraries to the date, which requires thinking of the habits of mind as a relatively new subject that deserves more investigation and comparison with other theories, such as previously described instructional learning.

\section{Conclusion}

This research explored the formation of habits of mind to improve student's thinking in class. Through a systematic review with meta-analysis, it was confirmed that habits of mind are related to the development of critical thinking skills, while its effect is varying from one habit to another and requires n-depth investigation for multiple disciplines. A further work in this direction assumes a field approach, where the facilities that attempted to implement a Framework concept would agree to disclose the relevance of methods deployed to the standards voiced. The design should assume taking a form of a mixed-method research as suggested be several prior researchers (i.e. Jacobson, 2014 or Goldstein \& Winner, 2012) to understand how cognitive research in psychology and management conforms to the educational approached undertaken by current representatives of science, school administration, and teachers. Additionally, it would be required to collect an unbiased opinion from education management professionals on the future perspectives and strategies to make the transition to the deployment of the concept of the habits of mind as more transparent as possible.

\section{References}

Astin, A. W. (1999). Student involvement: A developmental theory for higher education. Journal of College Student Development, 40(5), 518-529.

Augustine, T. A. (2014). Habits of the heart, habits of the mind: Teacher education for a global age (Doctoral Dissertation). Columbus, OH: The Ohio State University.

Borenstein, M., Hedges, L. V., Higgins, J. P. T., \& Rothstein, H. R. (2009). Introduction to meta-analysis. Chichester, UK: Wiley. https://doi.org/10.1002/9780470743386

Chahine, S. (2011). An investigation of educator's data habit of mind (Doctoral Dissertation). Toronto: University of Toronto, Canada.

Cohen, J. (1988). Statistical power analysis for the behavioral sciences (2nd ed.). Hillsdale, NJ: Erlbaum.

Costa, A., \& Kallick, B. (Eds.) (2000). Discovering and exploring habits of mind. Alexandria, VA: Association for Supervision and Curriculum Development.

Costa, A., \& Kallick, B. (Eds.) (2009). Habits of mind across the curriculum. Alexandria, VA: Association for Supervision and Curriculum Development.

Ellis, D., \& Murtha, M. (2014). Staging an essay: Play and playwriting for redirecting habits of mind. Journal of Basic Writing, 33(1), 74-102.

Fierke, K., Lui, K., Lepp, G., \& Baldwin, A. (2014). Teaching inclusive leadership through student-centered practices. Journal of the Academy of Business Education, 15(1), 51-65.

Fisher, E. L. (2017). A systematic review and meta-analysis of predictors of expressive-language outcomes 
among late talkers. Journal of Speech, 60(10), 2935-2948. https://doi.org/10.1044/2017_JSLHR-L-16-0310

Gardner, H. (1983). Frames of Mind: The theory of multiple intelligence. New York, NY: Basic Books

Gershman, S. J., Gerstenberg, T., Baker, C. L., \& Cushman, F. A. (2016). Plans, habits, and theory of mind. PLoS One, 11(9), 1-24. https://doi.org/10.1371/journal.pone.0162246

Glenewinkel, J. R. (2011). How do superintendents use the ISLLC Standards to evaluate principals? Or do they? (Unpublished Doctoral Dissertation). Pullman, WA: Washington State University, Pullman.

Goldstein, T. R., \& Winner, E. (2012). Enhancing empathy and theory mind. Journal of Cognition and Development, 13(1), 19-37. https://doi.org/10.1080/15248372.2011.573514

Groff, J. (2013). Expanding our frames of mind for education and the arts. Harvard Education Review, 83(1), 15-39. https://doi.org/10.17763/haer.83.1.kk34802147665819

Haber-Curran, P., \& Tillapaugh, D. (2015). Student-centered transformative learning in leadership education: An examination of the teaching and learning process. Journal of Transformative Education, 13(1), 65-84. https://doi.org/10.1177/1541344614559947

Hammer, S. J., \& Green, W. (2011). Critical thinking in a first year management unit: The relationship between disciplinary learning, academic literacy and learning progression. Higher Education Research \& Development, 30(3), 303-315. https://doi.org/10.1080/07294360.2010.501075

Higgins, J. P. T., \& Green, S. (Eds.). (2008). Cochrane handbook for systematic reviews of interventions. Chichester, UK: Wiley. https://doi.org/10.1002/9780470712184

Jacobson, L. (2014). From habits of mind to critical thinking: A study of student learning behaviors in a university great books general education course (Doctoral Dissertation). Philadelphia, PA: Temple University.

Johnson, K. (2013). Beyond standards: Disciplinary and national perspectives on habits of mind. College Composition and Communication, 64(3), 517-541.

King, K. (2013). Aftermath: Mathematical habits of mind. Math Horizons, 20(4), 34. https://doi.org/10.4169/mathhorizons.20.4.34

Kleinsteuber, A. (2014). The impact of professional development in the arts upon habits of mind and teacher efficacy (Doctoral Dissertation). Long Beach, CA: California State University.

Lammi, M.D., \& Denson, C.D. (2017). Modeling as an engineering habit of mind and practice. Advances in Engineering Education, 6(1), 1-27.

Liberati, A., Altman, D. G., Tetzlaff, J., Mulrow, C., Gøtzsche, P. C., Ioannidis, J. P. A., ... Moher, D. (2009). The PRISMA statement for reporting systematic reviews and meta-analyses of studies that evaluate healthcare interventions: Explanation and elaboration. The BMJ, 339, b2700. https://doi.org/10.1136/bmj.b2700

Loveland, T., \& Dunn, D. (2014). Teaching engineering habits of mind in technology education. Technology and Engineering Teacher, 73(8), 13-19.

Matsuura, R., Sword, S., Piecham, M.B., Stevens, G., \& Cuoco, A. (2013). Mathematical habits of mind for teaching: Using language in algebra classrooms. The Mathematics Enthusiast, 10(3), 735-776.

McPeck, J. (1984). Stalking beasts, but swatting flies: The teaching of critical thinking. Canadian Journal of Education, 9(1), 28-44. https://doi.org/10.2307/1494448

National Academy of Engineering. (2010). Standards for K-12 Engineering Education? Washington, DC: The National Academies Press

O’Neill, P., Adler-Kassner, L., Fleischer, C., \& Hall, A.-M. (2012). Symposium: On the framework for success in postsecondary writing. College English, 74(6), 520-533.

Petinou, K., \& Spanoudis, G. (2014). Early language delay phenotypes and correlation with later linguistic abilities. Folia Phoniatrica et Logopaedica, 66(1-2), 67-76. https://doi.org/10.1159/000365848

Rigby, J. (2014). Three Logics of Instructional Leadership. Educational Administration Quarterly, 50(4), 610-644. https://doi.org/10.1177/0013161X13509379

Sheridan, K.M. (2011). Envision and observe: Using the studio thinking framework for learning and teaching in digital arts. Mind, Brain, and Education, 5(1), 19-26. https://doi.org/10.1111/j.1751-228X.2011.01105.x

Stith, I., \& Roth, W. M. (2010). Teaching as mediation: The cogenerative dialogue and ethical understandings. 
Teaching and Teacher Education, 26, 363-370. https://doi.org/10.1016/j.tate.2009.09.008

Sullivan, P. (2012). Essential habits of mind for college readiness. College English, 74(6), 547-553.

Tolley, L., Johnson, L., \& Koszalka, T. (2012). An intervention study of instructional methods and student engagement in large classes in Thailand. International Journal of Education Research, 53, 381-393. https://doi.org/10.1016/j.ijer.2012.05.003

Walker, C. M., Wartenberg, T. E., \& Winner, E. (2013). Engagement in philosophical dialogue facilitates children's reasoning about subjectivity. Developmental Psychology, 49(7), 1338-1347. https://doi.org/10.1037/a0029870

Weiler, A. (2005). Information-seeking behavior in Generation Y students: Motivation, critical thinking, and learning theory. Journal of Academic Librarianship, 31(1), 46-53. https://doi.org/10.1016/j.acalib.2004.09.009

Williams, R. L. (1999). Operational definitions and assessment of higher-order cognitive constructs. Educational Psychology Review, 11(4), 411-427. https://doi.org/10.1023/A:1022065517997

Willingham, D. T. (2008). Critical thinking: Why is it so hard to teach? Arts Education Policy Review, 109(4), 21-32. https://doi.org/10.3200/AEPR.109.4.21-32

Zhu, C., \& Engels, N. (2014). Organizational culture and instructional innovations in higher education: Perceptions and reactions of teachers and students. Educational Management Administration \& Leadership, 42(1), 136-158. https://doi.org/10.1177/1741143213499253

\section{Copyrights}

Copyright for this article is retained by the author(s), with first publication rights granted to the journal.

This is an open-access article distributed under the terms and conditions of the Creative Commons Attribution license (http://creativecommons.org/licenses/by/4.0/). 